\title{
Effects of Inorganic Iron and Myoglobin on In Vitro Proximal Tubular Lipid Peroxidation and Cytotoxicity
}

\author{
R. A. Zager and C. A. Foerder \\ Department of Medicine, University of Washington, 98105; and the Fred Hutchinson Cancer Research Center, Seattle, Washington 98104
}

\begin{abstract}
Recent in vivo studies suggest that heme Fe causes proximal tubular lipid peroxidation and cytotoxicity, thereby contributing to the pathogenesis of myoglobinuric (Mgb) acute renal failure. Because hydroxyl radical $(\cdot \mathrm{OH})$ scavengers [dimethylthiourea (DMTU), benzoate, mannitol] can mitigate this injury, it is postulated that $\cdot \mathbf{O H}$ is a mediator of $\mathbf{M g b}$-induced renal damage. The present study has tested these hypotheses using an isolated rat proximal tubular segment (PTS) system. An equal mixture of $\mathrm{Fe}^{2+} / \mathrm{Fe}^{3+}$ (4 mM total), when added to PTS, caused marked cytotoxicity [as defined by lactate dehydrogenase (LDH) release] and lipid peroxidation [assessed by malondialdehyde (MDA) increments]. $\mathrm{Fe}^{2+}$ or $\mathrm{Fe}^{3+}$ alone each induced massive MDA elevations, but only $\mathrm{Fe}^{2+}$ caused cytotoxicity. Although both DMTU and benzoate decreased LDH release during the $\mathrm{Fe}^{2+} / \mathrm{Fe}^{3+}$ challenge, mannitol and GSH did not, despite equivalent reductions in - $\mathrm{OH}$ (gauged by the salicylate trap method). GSH and catalase (but not DMTU, benzoate, or mannitol) decreased MDA concentrations, suggesting the Fe-driven lipid peroxidation was more $\mathrm{H}_{2} \mathrm{O}_{2}$ than $\cdot \mathrm{OH}$ dependent. Deferoxamine totally blocked Fe-induced LDH release, even under conditions in which it caused an apparent increase in $\cdot \mathrm{OH}$ generation. Mgb paradoxically protected against Fe-mediated PTS injury, an effect largely reproduced by albumin. In conclusion, these data suggest that: (a) Fe can cause PTS lipid peroxidation and cytotoxicity by a non- $\cdot$ OHdependent mechanism; (b) Fe-mediated cytotoxicity and lipid peroxidation are not necessarily linked; and (c) Mgb paradoxically protects PTS against Fe-mediated injury, suggesting that: (i) Mgb Fe may require liberation from its porphyrin ring before exerting toxicity; and (ii) the protein residue may blunt the resulting injury. (J. Clin. Invest. 1992. 89:989-995.) Key words: hydroxyl radical $\bullet$ hydrogen peroxide • deferoxamine • glutathione $\bullet$ mannitol
\end{abstract}

\section{Introduction}

During wartime and with natural disasters, crush syndromeinduced acute renal failure (ARF) ${ }^{1}$ can assume epidemic pro-

Address correspondence and reprint requests to Richard A. Zager, M.D., Nephrology M-178, Fred Hutchinson Cancer Research Center, 1124 Columbia Street, Seattle, WA 98104.

Received for publication 19 July 1991 and in revised form 15 October 1991

1. Abbreviations used in this paper: apoMgb, Mgb without porphyrin; ARF, acute renal failure; DFO, deferoxamine; DHBA, dihydroxybenzoic acid; DMTU, dimethylthiourea; LDH, lactate dehydrogenase; MDA, malondialdehyde; Mgb, myoglobin; PTS, proximal tubule segments.

J. Clin. Invest.

(c) The American Society for Clinical Investigation, Inc.

0021-9738/92/03/0989/07 \$2.00

Volume 89, March 1992, 989-995 portions, overwhelming dialytic capabilities. Thus, interventions that can prevent or mitigate this form of renal damage are highly desirable. It is generally accepted that myoglobinuria is a critical pathogenetic component to this syndrome. However, because it is unclear how myoglobin (Mgb) exerts its nephrotoxicity, new and effective interventions remain to be defined.

In recent years, it has been hypothesized that Fe-mediated hydroxyl radical $(\cdot \mathrm{OH})$ production via the Haber Weiss/Fenton reactions with resultant oxidant stress is a dominant pathway for heme protein nephrotoxicity (1-3). The supporting evidence is as follows: $(a)$ the Fe chelator deferoxamine (DFO) can protect against experimental myohemoglobinuric ARF (13); (b) renal lipid peroxidation, as assessed by malondialdehyde (MDA) tissue assay, may occur after myohemoglobinuric renal damage $(1,2) ;(c)$ these MDA increments may be blunted by - OH scavengers and DFO therapy $(1,2)$; and $(d) \mathrm{Na}$ benzoate, dimethylthiourea, and mannitol, all $\cdot \mathrm{OH}$ scavengers, can confer in vivo functional and morphological protection (1, $4,5)$.

The above evidence implicating Fe-driven oxidant tissue stress as a mechanism for heme protein nephrotoxicity has been gathered exclusively by using whole-animal experiments. Although conclusions as to the presence or absence of protection with a particular agent are valid, the reasons for such results may not be as firm. This is because functional, morphological, or biochemical protection in in vivo experiments can reflect a complex interplay of hemodynamic, intraluminal, and primary tubular cell events. Thus, even though an antioxidant confers protection and decreases MDA, these results could be due to a nonoxidant action. For example, although mannitol protects against myohemoglobinuric $A R F$, recent data indicate that it does so by its diuretic, not its antioxidant, effect (4).

An alternative approach to in vivo studies of cellular mechanisms of injury is to eliminate vascular and intraluminal events by using in vitro systems. Since the proximal tubule is the principal target of cellular damage in myoglobinuric ARF, we have attempted to complement the above in vivo studies of heme protein/Fe nephrotoxicity by studying the responses of isolated proximal tubular segments (PTS) to both inorganic Fe and $\mathrm{Mgb}$ challenges. The following questions have been addressed: (a) Is Fe toxic to proximal tubular cells? $(b)$ If so, does this cytotoxicity directly correlate with tubular cell lipid peroxidation? (c) Can - OH scavengers consistently block Fe-mediated cytotoxicity and lipid peroxidation, thereby suggesting that - OH is responsible for that injury? $(d)$ Does DFO confer cytoprotection despite the fact that it can paradoxically accentuate - $\mathrm{OH}$ production (6)? (e) Does Mgb Fe, sequestered within a porphyrin ring, also produce oxidant tissue stress? The results of these studies form the basis of this report.

\section{Methods}

\section{PTS preparation}

PTS were isolated from cortices of 200-250-g male Sprague Dawley rats by using a protocol described by Weinberg et al. (7). This method, 
developed for isolating rabbit tubules, was followed exactly except for three modifications: $(a)$ before resecting the kidneys, they were perfused in vivo with ice-cold starting buffer (8) to obtain near-bloodless kidneys $(\sim 60 \mathrm{ml}$ of buffer injected retrograde into the abdominal aorta, efflux being permitted from a transected renal vein); (b) DFO (Ciba Pharmaceutical Co., Summit, NJ) was added to the collagenase digestion buffer $(0.66 \mathrm{mg} / \mathrm{ml})$ to chelate any free $\mathrm{Fe}$, e.g., generated during the digestion process. The DFO was removed after collagenase digestion by five washings of the resulting PTS preparation (8); and (c) the final PTS preparation was isolated by centrifugation through $32 \%$ Percoll, as previously described (8). The PTS were suspended (3-5 mg PTS protein $/ \mathrm{ml}$ ) in an incubation buffer containing the following (mM): $100 \mathrm{NaCl}, 2.1 \mathrm{KCl}, 25 \mathrm{NaHCO}_{3}, 2.4 \mathrm{KH}_{2} \mathrm{PO}_{4}, 1.2 \mathrm{CaCl}_{2}, 1.2$ $\mathrm{MgCl}_{2}, 1.2 \mathrm{MgSO}_{4}, 5$ glucose, $4 \mathrm{Na}$ lactate, $10 \mathrm{Na}$ butyrate, 1 alanine, and dextran $\mathrm{T}_{40} 0.6 \%$ and were gassed with $95 \% \mathrm{O}_{2} / 5 \% \mathrm{CO}_{2} \times 20 \mathrm{~min}$ (final pH 7.4). Each PTS preparation used cortices from one or two rats, the sample being split into three to six aliquots after rewarming from 4 to $36^{\circ} \mathrm{C}$ over $15 \mathrm{~min}$. All experiments were conducted under oxygenated conditions $\left(95 \% \mathrm{O}_{2} / \mathrm{CO}_{2}\right)$ at $36^{\circ} \mathrm{C}$ over a 45 -min period, maintaining the segments in suspension with a rotating $(120 \mathrm{rpm})$ water bath.

\section{Fe challenge and determination of PTS cytotoxicity/lipid peroxidation}

To assess $\mathrm{Fe}$ toxicity, a standardized $\mathrm{Fe}$ challenge with known $\cdot \mathrm{OH}$ generating capability was used unless otherwise stated. This challenge consisted of $2 \mathrm{mM} \mathrm{FeSO}_{4} / 2 \mathrm{mM} \mathrm{FeCl}_{3} / 10 \mathrm{mM}$ ADP (final buffer concentrations), the $\operatorname{ADP}\left(5: 1\right.$ ratio with $\left.\mathrm{Fe}^{3+}\right)$ being used to maintain the added $\mathrm{Fe}^{3+}$ (and any $\mathrm{Fe}^{3+}$ generated from $\mathrm{Fe}^{2+}$ ) in solution $(9,10)$. The amount of $\mathrm{Fe}$ used (total of $4 \mathrm{mM}$ ) was selected because preliminary studies demonstrated that this was the amount needed to induce consistent PTS toxicity over a 45-min incubation period. Cytotoxicity was defined as the percentage of lactate dehydrogenase (LDH) released from PTS into the buffer (over the 45-min incubation period) as done by other laboratories (e.g., references 7,11$)$. Lipid peroxidation was assessed at the end of the 45-min incubations by assaying the pelleted PTS for MDA content, using the thiobarbituric acid 532-510 nM subtraction method of Uchiyama and Mihara (12), as previously performed in this laboratory (4). The results were expressed as nanomoles MDA per milligram PTS protein (protein measured by the bicinchoninic acid assay; Pierce Chemical Co., Rockford, IL).

\section{Specific experimental protocols}

$\mathrm{Fe}$ challenge/no modifying agents. Each of five PTS preparations was divided into four aliquots: two were incubated with the standard Fe challenge and two were incubated without $\mathrm{Fe}$ addition. After $45 \mathrm{~min}$, the samples were analyzed for percent $L D H$ release and MDA concentrations.

To determine whether $\mathrm{Fe}^{2+}$ or $\mathrm{Fe}^{3+}$ was principally responsible for the observed changes, four sets of PTS were each divided into three aliquots and they were incubated for $45 \mathrm{~min}$ with either $4 \mathrm{mM} \mathrm{Fe}^{2+} / 20$ $\mathrm{mM}$ ADP , $4 \mathrm{mM} \mathrm{Fe}^{3+} / 20 \mathrm{mM}$ ADP (total Fe content kept the same as in the standard challenge), or PTS buffer only. After 45 min, percent LDH release and MDA concentrations were determined. Since $\mathrm{Fe}^{2+}$, but not $\mathrm{Fe}^{3+}$, was shown to be cytotoxic (see Results), the influence of the added ADP on this toxicity was assessed, since the ADP can presumably increase PTS adenylate pools. Thus, six PTS were challenged with $4 \mathrm{mM} \mathrm{Fe}^{2+}$ without ADP and the percent LDH released was compared with that seen in the above $\mathrm{Fe}^{2+} / \mathrm{ADP}$ challenge.

- $\mathrm{OH}$ scavenger addition. The following experiments assessed whether dimethylthiourea (DMTU), Na benzoate, and mannitol, documented $\cdot \mathrm{OH}$ scavengers, blunt $\mathrm{Fe}$-mediated cytotoxicity and lipid peroxidation. These three agents were chosen for study since each has been reported to confer in vivo cytoprotection against experimental models of Mgb-ARF $(1,4,5)$. Because of insufficient PTS yields to permit all three agents to be tested together, these experiments were divided into two phases: DMTU and benzoate effects and mannitol effects. First, five sets of PTS were divided into equal aliquots and subjected to: $(a)$ no addition; $(b)$ the standard $\mathrm{Fe}$ challenge; $(c)$ the Fe challenge +20
mM DMTU; or $(d)$ the Fe challenge $+20 \mathrm{mM}$ benzoate. After $45 \mathrm{~min}$, percent $L D H$ release and MDA concentrations were determined. Next the influence of mannitol was assessed. One aliquot from each of five PTS preparations was challenged with $(a)$ no addition, $(b)$ the standard $\mathrm{Fe}$ challenge, and $(c)$ the $\mathrm{Fe}$ challenge $+100 \mathrm{mM}$ mannitol. LDH release and MDA generation were measured as noted above.

Because the above $\cdot \mathrm{OH}$ scavengers could have exerted a non-Fedependent effect on either the PTS or on the LDH assay itself, $20 \mathrm{mM}$ benzoate; $20 \mathrm{mM}$ DMTU, or $100 \mathrm{mM}$ mannitol was added to non-Feexposed aliquots and the percent LDH released and MDA generated were compared with coincubated PTS aliquots without addition ( $n$ of 4-5 pairs for each agent). The adequate performance of the LDH assay was determined by ascertaining comparable total $\mathrm{LDH}$ values for the paired control and agent-exposed PTS aliquots.

Catalase, GSH, and SOD addition. To assess the effect of catalase, GSH, and SOD on Fe-induced cytotoxicity and lipid peroxidation, three paired aliquots, each from a separate PTS preparation, were exposed to $(a)$ no addition; $(b)$ the standard $\mathrm{Fe}$ challenge; or $(c)$ the $\mathrm{Fe}$ challenge plus one of the following: (i) $20 \mathrm{mM}$ GSH (Sigma Chemical Co., St. Louis, MO), (ii) bovine liver catalase $(58 \mu \mathrm{g} / \mathrm{ml}$, or $4,900 \mathrm{U}$; Sigma Chemical Co.), or (iii) SOD (bovine erythrocyte SOD; Sigma Chemical Co.; $60 \mu \mathrm{g} / \mathrm{ml}[n=2] ; 600 \mu \mathrm{g} / \mathrm{ml},[n=3])$. As noted above, the effect of these agents on the PTS, independent of Fe addition, was also assessed by comparing LDH release and MDA generation from non-Fe-exposed PTS with and without addition of these agents (n $=4-6$ pairs).

DFO addition. To determine DFO effects on Fe-mediated cytotoxicity/lipid peroxidation, six sets of PTS were prepared and each was divided into the following aliquots: $(a)$ no addition; $(b)$ DFO addition, $2 \mathrm{mM}$; (c) an Fe challenge (1 $\left.\mathrm{mM} \mathrm{Fe} \mathrm{F}^{2+} / 1 \mathrm{mM} \mathrm{Fe}^{3+} / 5 \mathrm{mM} \mathrm{ADP}\right)$; and (d) the Fe challenge $+2 \mathrm{mM}$ DFO. (Of note, DFO chelates $\mathrm{Fe}^{3+}$ on an equimolar basis, but it binds virtually no $\mathrm{Fe}^{2+}$ unless or until it undergoes oxidation to $\mathrm{Fe}^{3+}$. Thus, the DFO concentration used [ $\left.2 \mathrm{mM}\right]$ was sufficient to chelate all of the added $\mathrm{Fe}$, even assuming complete $\mathrm{Fe}^{2+}$ oxidation). Four of the six PTS preparations had a fifth aliquot prepared consisting of $2 \mathrm{mM} \mathrm{Fe}{ }^{2+} / 2 \mathrm{mM} \mathrm{Fe}^{3+} / 10 \mathrm{mM}$ ADP/2 mM DFO. Thus, in these experiments, DFO was present in an insufficient amount to chelate the entire Fe challenge. All aliquots were subjected to a 45min incubation followed by LDH and MDA assay.

$M g b$ challenge. To determine whether $\mathrm{Mgb} \mathrm{Fe}$, like inorganic $\mathrm{Fe}$, is capable of inducing cytotoxicity and lipid peroxidation, five PTS preparations were divided into four aliquots as follows: $(a)$ no addition; $(b)$ horse skeletal muscle Mgb addition (Sigma Chemical Co.; $40 \mathrm{mg} / \mathrm{ml}$ of PTS buffer simulating urine Mgb concentrations that can be found in Mgb-ARF) (4, 13); (c) Mgb addition, $40 \mathrm{mg} / \mathrm{ml}$ plus the standard $\mathrm{Fe}(2$ $\left.\mathrm{mM} \mathrm{Fe} \mathrm{F}^{2+} / 2 \mathrm{mM} \mathrm{Fe}{ }^{3+} / \mathrm{ADP}\right)$ challenge; and $(d)$ the Fe challenge alone. (The purpose of the latter 2 aliquots was to establish whether Mgb causes synergistic or additive cytotoxicity/lipid peroxidation in the presence of inorganic $\mathrm{Fe}$ ). The following aliquots were compared: $a$ vs. $b$ and $c$ vs. $d$. (The Mgb-containing samples, which contained no contaminating LDH, were diluted 1:5 to dilute out their color, thereby facilitating the spectrophotometric LDH assay [shown to be linear over at least a 1-10 dilution range].) For comparison with the Mgb data, the experiment described for aliquots $c$ and $d$ was repeated $(n=5)$, substituting BSA (Sigma Chemical Co.; $40 \mathrm{mg} / \mathrm{ml}$ ) for Mgb. (The BSA was first shown to contain no LDH activity and not to affect the LDH assay or PTS viability, as noted previously.)

To assess whether Mgb or BSA binds $\mathrm{Fe}$, potentially altering its ability to mediate free radical reactions, $25 \mu \mathrm{M} \mathrm{FeCl}_{3}$ was incubated with either $1 \mathrm{mg} / \mathrm{ml} \mathrm{Mgb}$ or BSA and catalytic Fe activity was determined by the bleomycin assay method, as described by Gutteridge and Hou (14). As a positive control, $25 \mu \mathrm{M} \mathrm{FeCl}_{3}$ was incubated with 100 $\mu \mathrm{M}$ DFO instead of a protein, and the assay was repeated.

\section{Quantifying $\cdot \mathrm{OH}$ production/scavenging by therapeutic agents}

To document $\cdot \mathrm{OH}$ production during the $\mathrm{Fe}$ challenge and to assess the ability of the various test compounds to scavenge $\cdot \mathrm{OH}$, the salicylate trap method was employed, as previously described in the litera- 
ture (15-17). In brief, this technique uses salicylate as a stable $\cdot \mathrm{OH}$ trap, specific hydroxylated salicylate by-products (2,3- and 2,5-dihydroxybenzoic acids; DHBA) being generated by this reaction. The latter are detected by HPLC with quantitation by electrochemical detection (16). Three sets of experiments were undertaken.

Determine $\cdot \mathrm{OH}(\mathrm{DHBA})$ generation with the standard Fe challenge and assess the capacity of the above test reactants to alter this reaction. The standard $\mathrm{Fe}$ challenge was added to a buffer consisting of the following (mM): $115 \mathrm{NaCl}, 2.1 \mathrm{KCl}, 25 \mathrm{NaHCO}_{3}, 2.4 \mathrm{KH}_{2} \mathrm{PO}_{4}, 1.2$ $\mathrm{MgSO}_{4}, 1.2 \mathrm{MgCl}_{2}, 1.2 \mathrm{CaCl}_{2}, \mathrm{pH} 7.4$, plus $10 \mathrm{Na}$ salicylate. To this reaction was added either one of the above test reactants ( $n=4-7 / \mathrm{com}$ pound) in the same concentrations discussed above or just $\mathrm{Fe}(n=13)$. After a 30-min incubation (a time at which the reaction was found to be complete), it was diluted 1:1 with the HPLC mobile phase (citrate/acetate buffer pH 3.6/19\% methanol) (16) and then the samples were immediately analyzed for the 2,3- and 2,5-DHBA by-products. The total (2,3- + 2,5-) DHBA concentrations were compared among the groups. To assess whether the effect of Mgb on DHBA production was porphyrin/porphyrin Fe dependent, the influence of $40 \mathrm{mg} / \mathrm{ml}$ apoMgb (Mgb stripped of its porphyrin ring) on Fe-mediated DHBA production was assessed. The apoMgb was prepared from the horse skeletal muscle Mgb stock, as previously described (18).

Prove that the $\mathrm{Fe}^{2+} / \mathrm{Fe}^{3+} / A D P$ challenge generates $\cdot \mathrm{OH}$ (DHBA) in the PTS system. Five sets of PTS were each divided into four aliquots: $(a)$ PTS alone; $(b)$ PTS $+10 \mathrm{mM}$ salicylate; $(c)$ PTS + the Fe challenge; and $(d)$ PTS + the Fe challenge $+10 \mathrm{mM}$ salicylate. After 45 min, LDH release and MDA and DHBA concentrations were determined.

Determine $\mathrm{Fe}^{2+}$ and $\mathrm{Fe}^{2+} / \mathrm{DFO}$ effects on $\cdot \mathrm{OH}(\mathrm{DHBA})$ production in the PTS system. Since $\mathrm{Fe}^{2+}$, but not $\mathrm{Fe}^{3+}$, is cytotoxic (see Results), the effect of $\mathrm{Fe}^{2+}$ on $\cdot \mathrm{OH}$ (DHBA) production, and the ability of DFO to influence it, was assessed. PTS were incubated with: $(a) 2.5 \mathrm{mM} \mathrm{Fe}^{2+}$ ( $\mathrm{FeSO}_{4}$; no ADP); (b) $2.5 \mathrm{mM} \mathrm{Fe}^{2+}+4 \mathrm{mM} \mathrm{DFO;} \mathrm{(c)} \mathrm{DFO} \mathrm{without}$ $\mathrm{Fe}^{2+}$; or $(d)$ no additions ( $n=4$ each from 4 PTS preparations). $1 \mathrm{mM}$ $\mathrm{Na}$ salicylate (a dose shown in preliminary studies to be sufficient to detect $\cdot \mathrm{OH}$ but which does not significantly affect $\mathrm{Fe}^{2+}$ cytotoxicity) was added to each aliquot. After $45 \mathrm{~min}$, DHBA generation and LDH release were assessed.

\section{Calculations and statistics}

All values are presented as mean \pm SEM. All LDH and MDA data were analyzed by paired Student's $t$ test. If more than two sets of paired data were compared, the Bonferroni correction was applied. Other statistical comparisons are as stated.
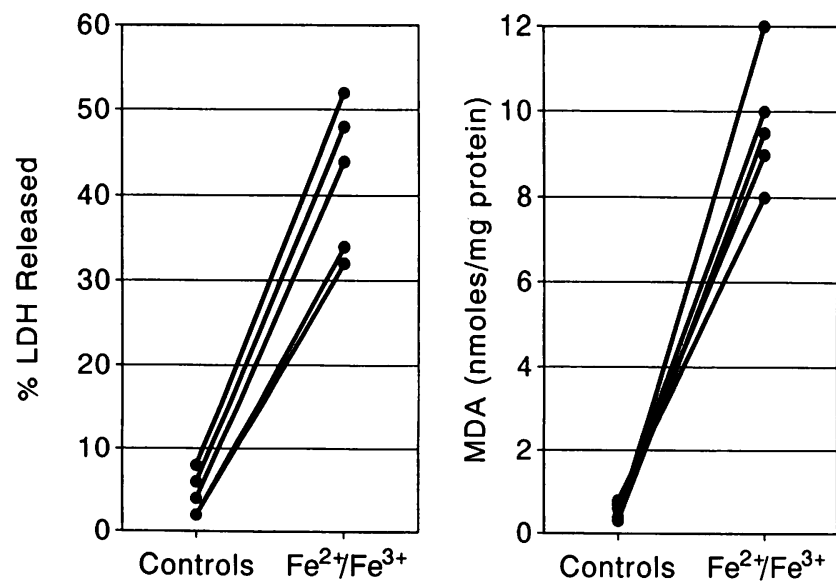

Figure 1. Percent LDH released (left) during a 45-min incubation in the absence of exogenous $\mathrm{Fe}$ (controls) or with the standard Fe challenge ( $\left.2 \mathrm{mM} \mathrm{Fe}^{2+} / 2 \mathrm{mM} \mathrm{Fe}^{3+} / \mathrm{ADP}\right)$ and the corresponding MDA concentrations at $45 \mathrm{~min}$ for these two groups (right). The Fe challenge caused marked increments in both LDH and MDA $(P<0.001)$.
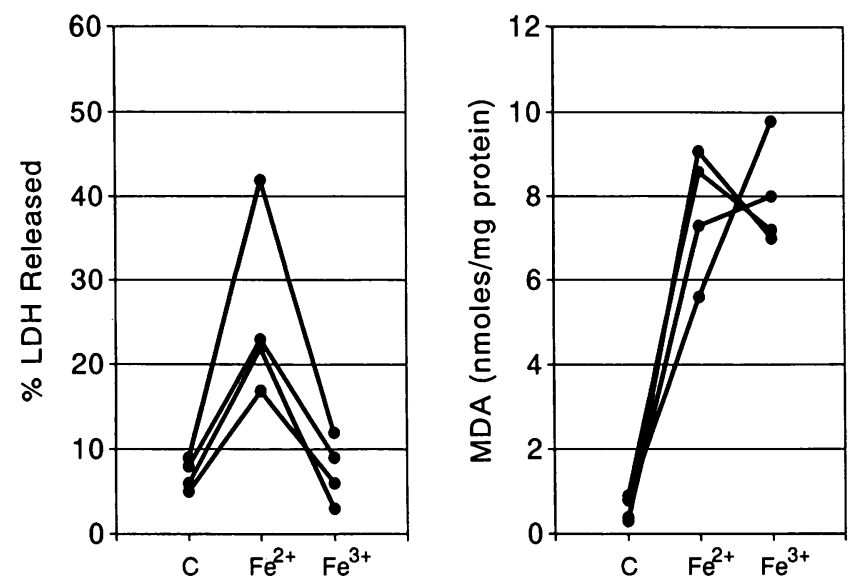

Figure 2. Percent LDH released over a 45-min incubation in the absence of an Fe challenge ( $C$, controls) or with either $4 \mathrm{mM} \mathrm{Fe}^{2+} / \mathrm{ADP}$ or $4 \mathrm{mM} \mathrm{Fe}^{3+} / \mathrm{ADP}$ addition. $\mathrm{Fe}^{2+}$ caused a significant increase in LDH release compared with the control PTS $(P<0.025)$, but $\mathrm{Fe}^{3+}$ failed to do so. However, both $\mathrm{Fe}^{2+}$ and $\mathrm{Fe}^{3+}$ caused marked and comparable MDA increments. Paired PTS aliquots are joined by lines.

\section{Results}

\section{Fe challenges}

The standard $\mathrm{Fe}^{2+} / \mathrm{Fe}^{3+}$ challenge caused marked cytotoxicity (Fig. 1): whereas the non-Fe-incubated PTS released $5 \pm 1 \%$ of their LDH over the 45-min incubation period, the Fe-exposed PTS had $41 \pm 4 \%$ LDH release over this same time period $(P$ $<0.001)$. This change coincided with a marked Fe-induced MDA increment compared with the non-Fe-exposed controls $(9.8 \pm 0.7$ vs. $0.4 \pm 0.04 \mathrm{nmol} / \mathrm{mg}$ protein, respectively; $P$ $<0.001$ ) (Fig. 1).

As shown in Fig. 2, incubation of PTS with only $\mathrm{Fe}^{2+} / \mathrm{ADP}$ caused both cytotoxicity and MDA increments $(P<0.025, P$ $<0.001$, respectively, compared with non-Fe-incubated controls). In contrast, $\mathrm{Fe}^{3+} / \mathrm{ADP}$ induced the same degree of MDA elevation as $\mathrm{Fe}^{2+}$, but it did not increase LDH release $(7.5 \pm 2 \%)$ compared with the coincubated non-Fe-exposed controls $(7 \pm 1 \%$; NS). The presence or absence of ADP did not affect this $\mathrm{Fe}^{2+}$-mediated toxicity (with ADP, $23 \pm 4 \%$; without ADP, $25 \pm 3 \%$ LDH release).

\section{- $\mathrm{OH}$ scavenger addition}

DMTU $(20 \mathrm{mM})$ and benzoate $(20 \mathrm{mM})$ substantially decreased Fe-mediated cytotoxicity, lowering the percent LDH release to just slightly above the $95 \%$ confidence band for nonFe-incubated PTS (Fig. 3, top). However, mannitol had no significant effect, despite the fact that a $100 \mathrm{mM}$ concentration was used. (Note: this $100-\mathrm{mM}$ dose was chosen because it was shown that a fivefold greater mannitol concentration was needed to reproduce the $\cdot \mathrm{OH}$ scavenging effect of $20 \mathrm{mM}$ DMTU and benzoate [see below]. Furthermore, initial studies with $20 \mathrm{mM}$ mannitol demonstrated that this dose also did not lessen Fe-mediated PTS cytotoxicity.) Despite the fact that DMTU and benzoate conferred striking cytoprotection (as did $10 \mathrm{mM}$ salicylate, discussed below), none of the $\cdot \mathrm{OH}$ scavengers had any apparent effect on Fe-mediated MDA generation (Fig. 3, bottom). Of note, DMTU, benzoate, and mannitol had no effect on LDH release or MDA generation during control incubations (NS by paired $t$ test compared with nonexposed coincubated controls). 


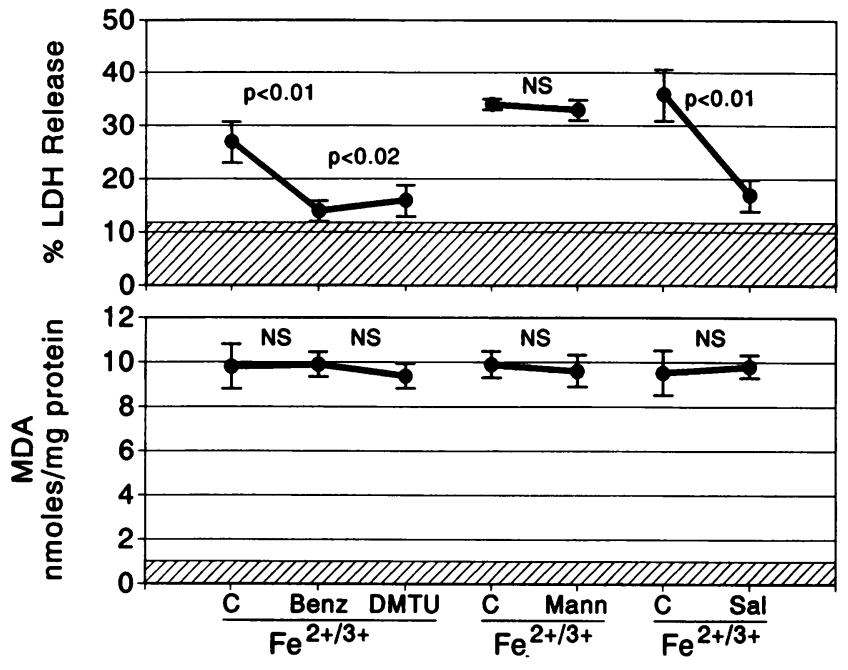

Figure 3. Effect of four $\cdot \mathrm{OH}$ scavengers on Fe-mediated $\mathrm{LDH}$ release (top) and MDA increments (bottom). $C$, controls (PTS incubated without an $\cdot \mathrm{OH}$ scavenger); Benz, $20 \mathrm{mM}$ Na benzoate; DMTU, 20 mM dimethylthiourea; Mann, $100 \mathrm{mM}$ mannitol; Sal, $10 \mathrm{mM} \mathrm{Na}$ salicylate. The lines join paired observations. The points represent mean values for the groups; bars, 1 SEM. All PTS aliquots were subjected to the standard $4 \mathrm{mM}$ Fe challenge. Benzoate, DMTU, and salicylate each decreased LDH release compared with their non- OH-scavenger-treated controls $(P<0.01, P<0.02, P<0.01$, respectively). However, mannitol did not have any cytoprotective effect. For comparison, the shaded area represents the $95 \%$ confidence interval for percent LDH released from PTS incubated for $45 \mathrm{~min}$ without Fe exposure. Despite the fact that benzoate, DMTU, and salicylate decreased LDH release, none of these agents decreased the Fe-mediated MDA increments. The shaded area represents the $95 \%$ confidence interval for MDA concentrations for PTS incubated for 45 min without $\mathrm{Fe}$ exposure. Paired data are joined by lines.

\section{Catalase, GSH, SOD, and DFO addition}

As shown in Fig. 4, catalase and GSH conferred no cytoprotection against the standard Fe challenge, as assessed by LDH release. However, catalase and GSH significantly decreased Femediated MDA generation (Fig. 4, bottom). SOD addition had no effect on LDH release or MDA generation in the presence or absence of the Fe challenge (although it remains possible that the SOD could have been inactivated during the PTS incubation). In the absence of the Fe challenge, $20 \mathrm{mM}$ GSH significantly decreased LDH release over the 45-min incubation period (GSH-exposed PTS, $3 \pm 0.4 \%$; no addition controls, $5 \pm 0.3 \% ; P<0.003 ; n=6$ pairs) without affecting MDA concentrations. Catalase had no Fe-independent effect.

DFO (2 mM) conferred virtually complete cytoprotection against the $1 \mathrm{mM} \mathrm{Fe} e^{2+} / 1 \mathrm{mM} \mathrm{Fe}^{3+} / \mathrm{ADP}$ challenge (Fig. $5 ; P$ $<0.01)$. However, the MDA increment, although significantly blunted $(P<0.01)$, was not eliminated; the values still remained $\sim 20$-fold higher than those in the non-Fe-exposed PTS (Fig. 5). Addition of excess $\mathrm{Fe}\left(2 \mathrm{mM} \mathrm{Fe}^{2+} / 2 \mathrm{mM} \mathrm{Fe}^{3+}\right)$ eliminated the cytoprotective and MDA-lowering effect of DFO ( $2 \mathrm{mM})$. In the absence of Fe, DFO had no effect on LDH/MDA values compared with control-incubated aliquots.

\section{Mgb challenge}

In the absence of the Fe challenge. As shown in Fig. 6, when the PTS were incubated with $40 \mathrm{mg} / \mathrm{ml}$ of Mgb, it conferred cytoprotection, decreasing the LDH release that normally occurs during $45 \mathrm{~min}$ of just $\mathrm{O}_{2} / \mathrm{CO}_{2}$ incubation $(6.4 \pm 0.4$ vs.

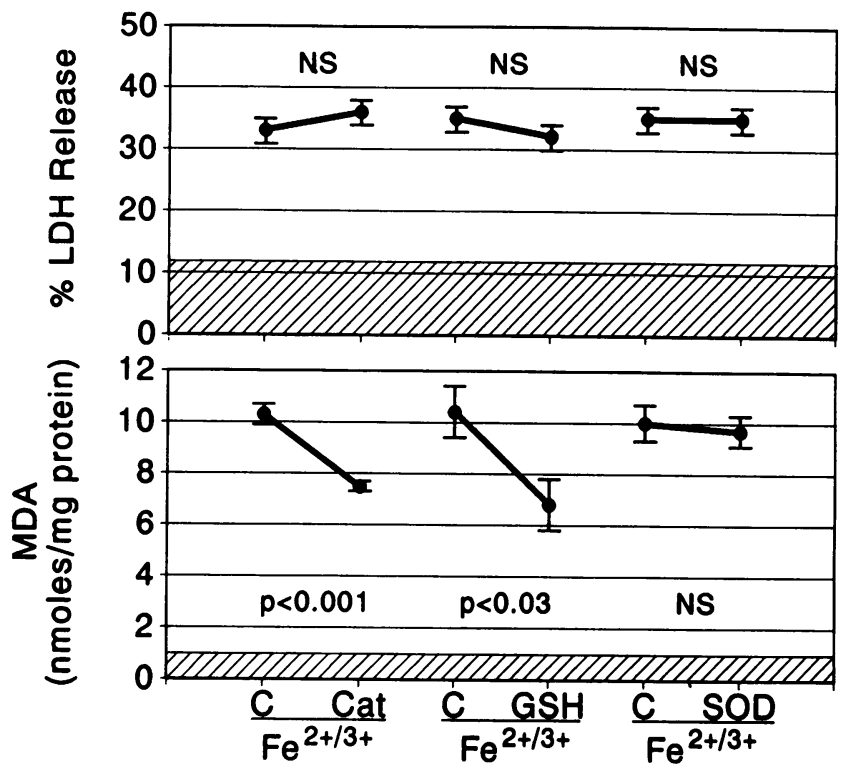

Figure 4. Percent LDH released and MDA concentrations in $\mathrm{Fe}^{2+}$ / $\mathrm{Fe}^{3+} / \mathrm{ADP}$-challenged PTS with and without catalase (Cat), glutathione $(G S H)$, or superoxide dismutase $(S O D)$. $C$, control PTS (challenged with $\mathrm{Fe}$ in the absence of an antioxidant). Paired data (mean \pm SEM) are joined by lines. The $95 \%$ confidence intervals for percent $L D H$ release and MDA concentrations in non-Fe-exposed PTS aliquots are depicted by the shaded areas. Catalase and GSH significantly reduced MDA concentrations but they conferred no cytoprotection, as assessed by LDH release. SOD had no discernible effects.

$2.1 \pm 0.8 \% ; P<0.015)$. The amount of MDA generated over this 45-min incubation was also significantly reduced $(0.78 \pm 0.25$ vs. $0.44 \pm 0.21 \mathrm{nmol} / \mathrm{mg}$ protein; $P<0.001)$.

In the superimposed standard Fe challenge. Mgb addition also protected the PTS against the standard Fe challenge. LDH release was decreased from $37 \pm 5$ to $20 \pm 2 \%(P<0.05)$ and
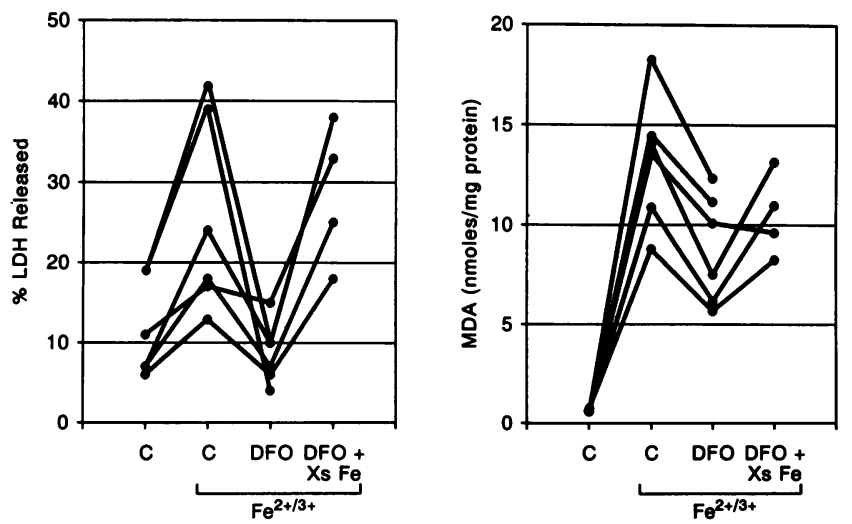

Figure 5. DFO effects on LDH release and MDA generation. $C$, control PTS incubated with and without an Fe challenge. Two DFO protocols were tested: the first assessed the influence of $2 \mathrm{mM}$ DFO added to PTS challenged with $2 \mathrm{mM} \mathrm{Fe}\left(1 \mathrm{mM} \mathrm{Fe}^{2+} / 1 \mathrm{mM} \mathrm{Fe}^{3+} /\right.$ ADP); the second gtoup had $2 \mathrm{mM}$ DFO added but excess ( $X \mathrm{~s}) \mathrm{Fe}(2$ $\mathrm{mM} \mathrm{Fe}{ }^{2+} / 2 \mathrm{mM} \mathrm{Fe}^{3+} / \mathrm{ADP}$ ) was used, thereby exceeding the chelating capacity of DFO. DFO totally blocked the LDH release induced by $1 \mathrm{mM} \mathrm{Fe}^{2+} / 1 \mathrm{mM} \mathrm{Fe}^{3+}(P<0.01)$, but it only slightly (but significantly; $P<0.01$ ) attenuated the lipid peroxidation. Addition of excess $\mathrm{Fe}$ abrogated both of these beneficial DFO effects. Individual sets of paired data are joined by lines. 

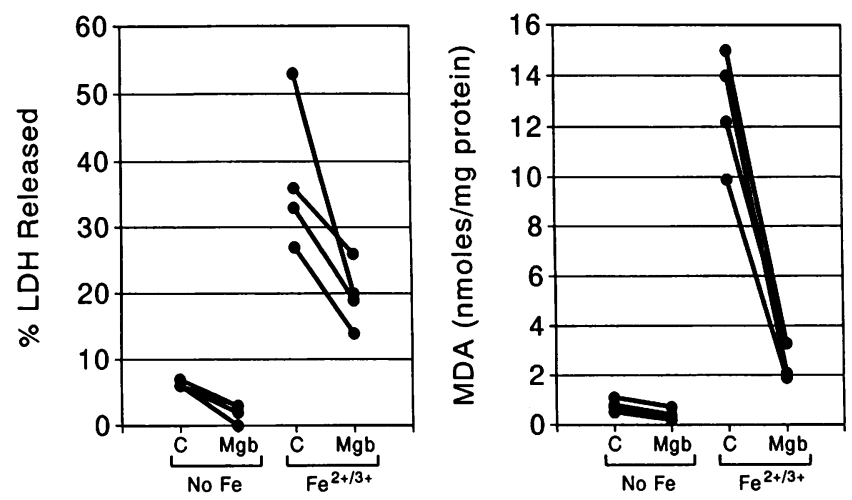

Figure 6. Mgb effects on PTS LDH release and MDA concentrations in the presence and absence of a $2 \mathrm{mM} \mathrm{Fe}^{2+} / 2 \mathrm{mM} \mathrm{Fe}^{3+} / \mathrm{ADP}$ challenge. $C$, control PTS (not subjected to Mgb addition). Mgb significantly blunted LDH release and MDA generation during both non-Fe and Fe incubation (statistics as per text). Individual PTS aliquot values are presented with paired data connected by lines.

MDA was reduced by $78 \%$ (from $12.9 \pm 2.4$ to $2.9 \pm 0.7 \mathrm{nmol}$ / mg protein; $P<0.005$ ) (Fig. 6). The ability of Mgb to mitigate $\mathrm{Fe}$-mediated cytotoxicity was not Mgb specific, since BSA also decreased $\mathrm{LDH}$ release during the $\mathrm{Fe}$ challenge (BSA + Fe, $21 \pm 7 \%$; Fe alone, $35 \pm 7 \% ; P<0.01$ ). However, unlike $\mathrm{Mgb}$, BSA did not decrease MDA generation (BSA + Fe, $9.0 \pm 1.1 ; \mathrm{Fe}$ alone, $8.6 \pm 1.2 \mathrm{nmol} \mathrm{MDA} / \mathrm{mg}$ protein; NS). Neither Mgb nor BSA decreased catalytic Fe activity, as assessed by the bleomycin assay. In fact, both proteins tended to increase it. In contrast, DFO completely inhibited the Fe-bleomycin reaction, indicating Fe chelation.

\section{Salicylate trap assessment of $\cdot \mathrm{OH}$ production/scavenging}

In the absence of PTS. Incubation of salicylate without Fe caused no detectable 2,3- or 2,5-DHBA production. However, salicylate + the standard Fe challenge caused striking DHBA increments (total, $156 \pm 6 \mathrm{nmol} / \mathrm{ml} ; 95 \%$ confidence band, $114-196 \mathrm{nmol} / \mathrm{ml}$ ). Approximately 40 and $60 \%$ of this total DHBA represented 2,3- and 2,5-DHBA, respectively. As depicted in Fig. 7, DMTU, benzoate, mannitol, GSH, and catalase each significantly lowered DHBA production, the mean for each falling below the $95 \%$ confidence band. SOD was without effect. Although the mean DHBA result with DFO addition was not below the $95 \%$ confidence band for the unopposed $\mathrm{Fe}$ challenge, a direct comparison of the $\mathrm{Fe}$ alone versus $\mathrm{Fe}$ + DFO results revealed a significant difference by unpaired Student's $t$ test $(156 \pm 6$ vs. $120 \pm 10 \mathrm{nmol} / \mathrm{ml}$, respectively; $P$ $<0.01$ ). Mgb, apoMgb, and BSA each markedly lowered DHBA production (Fig. 7).

In the presence of PTS $+\mathrm{Fe}^{2+} / \mathrm{Fe}^{3+} / A D P$. Salicylate addition to PTS in the absence of the standard Fe challenge yielded only $3 \pm 0.4 \mathrm{nmol} / \mathrm{ml}$ total DHBA; in contrast, in the presence of the standard Fe challenge, $71 \pm 1 \mathrm{nmol} / \mathrm{ml} \mathrm{DHBA}$ resulted $(P$ $<0.001$ ). The $10 \mathrm{mM}$ salicylate addition also significantly decreased percent LDH release, but it did not alter MDA generation (Fig. 3).

$\mathrm{Fe}^{2+}$ effects on PTS $\cdot \mathrm{OH}$ production \pm DFO. In striking contrast to the above, $2.5 \mathrm{mM} \mathrm{Fe}{ }^{2+}$ (no Fe ${ }^{3+} / \mathrm{ADP}$ addition) caused no excess DHBA production $\left(\mathrm{Fe}^{2+}, 2 \pm 0.3\right.$; controls, $3 \pm 0.3 \mathrm{nmol} / \mathrm{ml})$. Nevertheless, $\mathrm{Fe}^{2+}$ induced cytotoxicity $(31 \pm 2 \%$ controls, $5 \pm 1 \%$ LDH release; $P<0.002)$. DFO in the absence of $\mathrm{Fe}^{2+}$ caused no excess DHBA generation $(4 \pm 0.3$ $\mathrm{nmol} / \mathrm{ml})$ or toxicity $(6 \pm 0.2 \% \mathrm{LDH}$ release). When added to the $\mathrm{Fe}^{2+}$ challenge, DFO eliminated toxicity of $\mathrm{Fe}(6 \pm 2 \% \mathrm{LDH}$ release) despite increasing DHBA $(\cdot \mathrm{OH})$ generation $\left(\mathrm{Fe}^{2+}\right.$ + DFO, 10 $\pm 0.4 ;$ Fe alone, $2 \pm 0.3 \mathrm{nmol} / \mathrm{ml} ; P<0.005)$.

\section{Discussion}

In 1988, Paller and Hedlund (19) indicated that Fe could be a potent cytotoxic factor in experimental tubular damage because DFO administration could protect against, and $\mathrm{FeCl}_{3}$ EDTA infusion could worsen, postischemic ARF. That several groups (1-3) have reported DFO-mediated protection against myohemoglobinuric ARF further supports this view. Because -OH scavengers can also blunt myohemoglobinuric renal injury $(1,4)$, it has been postulated that heme protein nephrotoxicity may be, in part, $\cdot \mathrm{OH}$ dependent. In this study, we have attempted to confirm these conclusions concerning heme protein nephrotoxicity by demonstrating $\mathrm{Fe}$-mediated $\cdot \mathrm{OH}$ production, cytotoxicity, and lipid peroxidation in isolated PTS. Using a known $\cdot \mathrm{OH}$-generating system $\left(\mathrm{Fe}^{2+} / \mathrm{Fe}^{3+} / \mathrm{ADP}\right)$, significant $\mathrm{LDH}$ release, $\cdot \mathrm{OH}$-dependent salicylate by-product generation, and MDA increments were all observed, supporting each of the above conclusions. It could be argued that the massive Fe concentrations we used, necessary to induce cytotoxicity in our system, grossly exceed renal Fe levels achievable in in vivo states. However, in the glycerol ARF model, urinary $\mathrm{Mgb}$ concentrations of $30-50 \mathrm{mg} / \mathrm{ml}$ can occur (4), equating to a 2-3 mM Fe load. Thus, it is theoretically possible that the $\mathrm{Fe}$ concentrations needed to induce in vitro PTS killing could be achieved during in vivo pathological states.

The second goal of this study was to confirm that Fe-induced cytotoxicity and lipid peroxidation are, in fact, closely linked. The relevance of this issue is that parallel changes in lipid peroxidation (MDA) and in the extent of renal damage have been widely used to imply an oxidant mechanism of injury in in vivo models of ARF (e.g., 1-4, 20, 21). On first

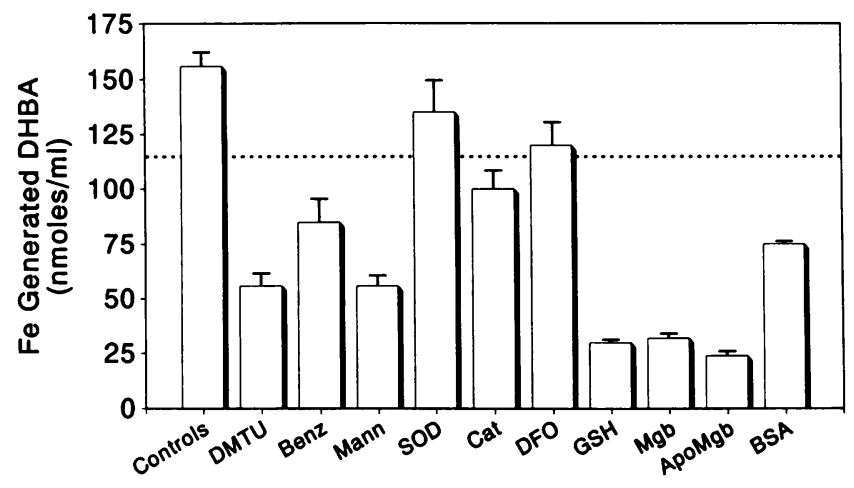

Figure 7. Dihydroxybenzoic acid $(D H B A)$ generated in response to the $2 \mathrm{mM} \mathrm{Fe}^{2+} / 2 \mathrm{mM} \mathrm{Fe}^{3+} / \mathrm{ADP}$ challenge. For ease of presentation, the sum of 2,3- and 2,5-DHBA is presented, the values representing mean \pm SEM. Controls, no addition (other than the Fe challenge); DMTU, $20 \mathrm{mM}$ dimethylthiourea; Benz, $20 \mathrm{mM}$ Na benzoate; Mann, $100 \mathrm{mM}$ mannitol; SOD, $600 \mu \mathrm{g} / \mathrm{ml}$ superoxide dismutase; $C a t, 58$ $\mu \mathrm{g} / \mathrm{ml}$ catalase; $D F O, 5 \mathrm{mM}$ deferoxamine; $G S H, 20 \mathrm{mM}$ glutathione; $M g b, 40 \mathrm{mg} / \mathrm{ml}$ myoglobin; apoMgb, $40 \mathrm{mg} / \mathrm{ml}$ myoglobin without porphyrin; and $B S A, 40 \mathrm{mg} / \mathrm{ml}$ bovine serum albumin. The dotted line represents the lower limit of the $95 \%$ confidence interval for DHBA production by the controls (Fe addition, no antioxidants). All of the agents except SOD and DFO lowered DHBA to below this value (indicating decreased $\cdot \mathrm{OH}$ production and/or $\cdot \mathrm{OH}$ scavenging. 
inspection, our data suggest that such a linkage exists, since the unopposed $\mathrm{Fe}^{2+} / \mathrm{Fe}^{3+}$ challenge caused marked and parallel changes in LDH release and MDA generation (Fig. 1). However, additional experiments seemingly dissociate these two findings. First, equimolar $\mathrm{Fe}^{2+}$ and $\mathrm{Fe}^{3+}$ caused virtually identical MDA increments, but only $\mathrm{Fe}^{2+}$ induced $\mathrm{LDH}$ release (Fig. 2). Second, DMTU, benzoate, and salicylate conferred marked cytoprotection, but none blunted the MDA changes (Fig. 3). Third, catalase and GSH each decreased MDA, but neither influenced LDH release (Fig. 4); and fourth, although DFO lowered both $\mathrm{LDH}$ release and MDA concentrations, these reductions were not equivalent: whereas DFO conferred essentially complete cytoprotection, MDA remained in 20-fold excess of normal concentrations (Fig. 5). Given these findings, it is tempting to postulate that cytotoxicity and lipid peroxidation are not pathogenetically linked. However, although this remains an intriguing possibility, it is also possible that although lipid peroxidation is not sufficient to cause cell killing, it could be a prerequisite for cytotoxicity to occur. Whatever the link is between lipid peroxidation and cytotoxicity, one point seems clear: caution is warranted when interpreting MDA concentrations in in vivo experiments since they need not correlate with, nor necessarily have pathogenetic relevance to, the evolution of lethal cellular injury.

The third goal of this study was to confirm in vivo suggestions that $\cdot \mathrm{OH}$ scavengers protect against Fe-induced proximal tubular damage. If so, this would support the hypothesis that $\cdot \mathrm{OH}$ is a critical mediator of this reaction, as previously inferred in the literature. As a prelude to these studies, effective and equivalent $\cdot \mathrm{OH}$ scavenging dosages of DMTU, benzoate, and mannitol were defined in the Fe-salicylate reaction. When these equivalent doses were subsequently applied to the PTS system, DMTU and benzoate almost totally blocked $\mathrm{Fe}^{2+} /$ $\mathrm{Fe}^{3+}$-mediated LDH release (Fig. 3), supporting conclusions from in vivo studies that these agents mitigate Fe-driven tubular damage (1). However, mannitol and GSH, which induced comparable decrements in DHBA production, were devoid of any apparent cytoprotective effects (Fig. 4), suggesting that DMTU and benzoate may have protected via a non- $\cdot \mathrm{OH}-$ dependent mechanism. For example, DMTU and benzoate (and closely related salicylate) can weakly complex Fe, potentially interfering with its bioavailability. Alternatively, they could have exerted a primary cytoprotective effect. Whatever the exact mechanism, the data underscore that ". OH-scavenging effects" and "protective effects" are not necessarily synonymous. Since the principal evidence implicating $\cdot \mathrm{OH}$ as a mediator of Mgb-induced tubular cell killing in vivo is benzoate/ DMTU-mediated cytoprotection and reductions in MDA, the failure of GSH and mannitol to decrease $\mathrm{LDH}$ release despite lowering - OH (DHBA) and the inability of DMTU, benzoate, or mannitol to blunt MDA generation, leaves this conclusion open to question. That catalase and GSH decreased MDA generation, but that benzoate, mannitol, and DMTU did not, suggests that $\mathrm{H}_{2} \mathrm{O}_{2}$, rather than $\cdot \mathrm{OH}$, was a more important determinant of the $\mathrm{Fe}^{2+} / \mathrm{Fe}^{3+}$-mediated proximal tubular lipid peroxidation in our system.

Since GSH (or, more specifically, its constituent amino acid, glycine) can protect PTS against a broad range of insults (e.g., 22-24), it was surprising that $20 \mathrm{mM}$ GSH did not lessen $\mathrm{Fe}$ cytotoxicity in these studies. However, it should be noted that we employed a much higher GSH concentration ( $20 \mathrm{mM})$, raising the possibility that the GSH may have exerted an independent toxic effect. However, that $20 \mathrm{mM} \mathrm{GSH}$ actually im- proved PTS viability under control incubation conditions seemingly excludes this possibility. Two additional considerations are that GSH is sufficiently altered by apparent $\cdot \mathrm{OH}$ scavenging (Fig. 7) so that it cannot exert a protective effect, and that Fe may cause a type of injury against which GSH cannot protect. Additional experimentation is needed to help resolve these (and other) possibilities.

The fourth goal of this study was to determine whether DFO can prevent the cytotoxic and lipid peroxidative effects of Fe. Although several in vivo studies have demonstrated that DFO can mitigate myohemoglobinuric ARF (1-3), one report suggested that it might do so by enhancing urinary heme protein excretion rather than by conferring direct cytoprotection (3). Klebanoff et al. (6) showed that, under selected circumstances, DFO can paradoxically increase $\mathrm{Fe}^{2+}$-mediated $\cdot \mathrm{OH}$ production, which further calls this issue into question. The results of our PTS study demonstrate that DFO can indeed protect PTS against Fe cytotoxicity and decrease lipid peroxidation, strongly supporting the data of Shah and Walker (1) and Paller (2). However, the data suggest that this protective effect may not necessarily be due to decreased $\cdot \mathrm{OH}$ generation. First, DFO, when added to a strong $\cdot \mathrm{OH}$-generating system $\left(\mathrm{Fe}^{2+} / \mathrm{Fe}^{3+} / \mathrm{ADP}\right)$, had a seemingly trivial impact on $\cdot \mathrm{OH}$ (DHBA) production (Fig. 7), despite the fact that the DFO blocked the cytotoxic effects of Fe; second, when a $2.5 \mathrm{mM}$ $\mathrm{Fe}^{2+}$ challenge was used, cytotoxicity occurred in the absence of apparent $\cdot \mathrm{OH}$ generation; and third, when DFO was added to this $\mathrm{Fe}^{2+}$ challenge, it conferred complete protection despite the fact that it appeared to increase $\cdot \mathrm{OH}$ production, consistent with the findings of Klebanoff et al. (6). Thus, these data, along with the failure of mannitol and GSH to confer cytoprotection, strongly suggest that $\cdot \mathrm{OH}$ was not the key factor in Fe-mediated PTS toxicity, at least in these experiments. Thus a more attractive hypothesis is that DFO accelerates $\mathrm{Fe}^{2+} \rightarrow \mathrm{Fe}^{3+}$ autoxidation (6), thereby rapidly eliminating cytotoxic $\mathrm{Fe}^{2+}$ (Fig. 2) from the PTS reaction. Because DFO addition to $\mathrm{Fe}^{2+}$ containing PTS buffer caused the immediate formation of a bright orange color, indicating $\mathrm{Fe}^{3+} / \mathrm{DFO}$ complex (feroxamine) formation, this possibility is directly supported.

The final goal of this study was to explore whether Mgb, like Fe, is cytotoxic to PTS. Thus, $40 \mathrm{mg} \mathrm{Mgb} / \mathrm{ml}$, mimicking urinary Mgb concentrations in the glycerol ARF model, was added to PTS. Paradoxically, a striking cytoprotective influence was observed: Mgb blunted the slight lipid peroxidation and $\mathrm{LDH}$ release that occurred with just $\mathrm{O}_{2} / \mathrm{CO}_{2}$ incubation, and it decreased the cytotoxicity and massive MDA increments that resulted from inorganic Fe addition. Several conceivable explanations for this result exist. First, that Mgb blunted DHBA production (Fig. 7) indicates that it can either decrease - $\mathrm{OH}$ generation or scavenge $\cdot \mathrm{OH}$, thereby limiting cytotoxicity. However, the above considerations would seem to rule out an $\cdot \mathrm{OH}$-dependent mechanism. Second, Mgb's porphyrin ring conceivably could have chelated the Fe challenge, thereby exerting a "DFO-like" effect. However, because Mgb is normally Fe saturated; BSA reproduced the cytoprotective effect of Mgb; stripping Mgb of its porphyrin ring (apoMgb) did not diminish its ability to decrease DHBA generation; and Mgb did not decrease catalytic Fe activity as assessed by the bleomycin assay, this possibility seems to be excluded. Third, and most likely, is that $\mathrm{Mgb}$ weakly complexed Fe via a non-porphyrin-dependent reaction, thereby decreasing its bioavailability to PTS. For example, if $\mathrm{Fe}$ toxicity were dependent on its binding to PTS, provision of extraneous protein "binding sites" might confer 
protection. Regardless of the mechanism, the protective influence of $\mathrm{Mgb}$ raises two important possibilities: first, if $\mathrm{Mgb} \mathrm{Fe}$ possesses a direct toxic effect, a prerequisite for it may be its liberation from the porphyrin ring; and second, if Fe liberation occurs, the protein residue might mitigate the resulting toxicity. In support of this second possibility is a previous observation from our laboratory that intrarenal Mgb accumulation may actually decrease, rather than increase, renal cortical MDA concentrations in the glycerol ARF model (4).

In summary, the present study confirms that $\mathrm{Fe}$ induces both renal tubular lipid peroxidation and cytotoxicity. However, the data also suggest that these two processes are not directly linked. Both DMTU and benzoate can prevent tubular cell injury, supporting conclusions derived from in vivo experiments. However, that equally effective protocols for decreasing - OH (mannitol, GSH administration) did not reproduce this cytoprotection suggests that the DMTU/benzoate effects may have occurred via a non-- OH-dependent mechanism (e.g., Fe complexing, thereby decreasing its bioavailability). Catalase and GSH blunted Fe-mediated MDA increments, but DMTU, benzoate, and mannitol did not. This suggests that the observed Fe-mediated PTS lipid peroxidation was more $\mathrm{H}_{2} \mathrm{O}_{2}$ than $\cdot \mathrm{OH}$ dependent. DFO blocks Fe-mediated PTS cytotoxicity, again consistent with in vivo observations. However, it appears to do so not by decreasing $\cdot \mathrm{OH}$ generation (in fact, it may increase it) but rather by accelerating $\mathrm{Fe}^{2+} \rightarrow \mathrm{Fe}^{3+}$ autoxidation, thereby eliminating cytotoxic $\mathrm{Fe}^{2+}$ from the PTS media. Surprisingly, Mgb (and BSA) can protect against Fe-mediated PTS toxicity. This suggests that, if in vivo Mgb toxicity is Fe dependent, Fe liberation from its porphyrin ring may be a prerequisite for this toxicity to occur, and that the residual protein may serve to mitigate the resulting Fe-mediated damage.

Lastly, we wish to caution that our results have no proven relevance to in vivo Mgb-ARF. Of note, the nature, concentrations, and routes of Fe delivery to in vivo tubular cells may differ markedly from what existed in our in vitro system. Thus, although the present study suggests that $\cdot \mathrm{OH}$ is not the critical mediator of Fe toxicity in our system, this does not exclude a very different mechanism of injury in vivo or in different in vitro systems. Obviously, additional in vivo and in vitro studies are required to help resolve this issue.

\section{Acknowledgments}

We thank Dr. Seymour Klebanoff for his helpful comments during the course of these studies. D. Gmur kindly performed DHBA analyses. We thank B. Schimpf and C. Bredl for their technical assistance and D. Jensen for her expert secretarial support.

This work was funded by grants from the National Institutes of Health (DK-38432 and GM-43686).

\section{References}

1. Shah, S. V., and P. D. Walker. 1988. Evidence suggesting a role for hydroxyl radical in glycerol-induced acute renal failure. Am. J. Physiol. 255:F438-F443.

2. Paller, M. S. 1988. Hemoglobin- and myoglobin-induced acute renal failure in rats: role of iron in nephrotoxicity. Am. J. Physiol. 255:F539-F544.

3. Zager, R. A., and L. M. Gamelin. 1989. Pathogenetic mechanisms in experimental hemoglobinuric acute renal failure. Am. J. Physiol. 256:F446-F455.

4. Zager, R. A., C. A. Foerder, and C. Bredl. 1991. The influence of mannitol on myoglobinuric acute renal failure: functional, biochemical, and morphologic assessments. J. Am. Soc. Nephrol. 2:848-855.

5. Parry, W. L., J. A. Schaefer, and C. B. Meuller. 1963. Experimental studies of acute renal failure. I. Protective effect of mannitol. J. Urol. 89:1-6.

6. Klebanoff, S. J., A. M. Wallersdorph, and H. Rosen. 1989. Oxygen-based free radical generation by ferrous ions and deferoxamine. J. Biol. Chem. 264:19765-19771.

7. Weinberg, J. M., J. A. Davis, M. Abarzua, and T. Rajan. 1987. Cytoprotective effects of glycine and glutathione against hypoxic injury to tubules. J. Clin. Invest. 80:1446-1454.

8. Zager, R. A., D. J. Gmur, C. R. Bredl, and M. J. Eng. 1991. Temperature effects on ischemic and hypoxic renal proximal tubular injury. Lab. Invest. 64:766-776.

9. Gutteridge, J. M. C. 1988. Lipid peroxidation: some problems and concepts. In Oxygen Radicals in Tissue Injury. B. Halliwell, editor. Allen Press, Lawrence, KA. 9-19.

10. Bucher, J. R., M. Tien, and S. D. Aust. 1983. The requirement for ferric in the initiation of lipid peroxidation by chelated ferrous iron. Biochem. Biophys. Res. Commun. 111:776-784.

11. Mandell, L. S., R. G. Schnellmann, and W. R. Jacobs. 1990. Intracellular glutathione in the protection from anoxic injury in renal proximal tubules. $J$. Clin. Invest. 85:316-324.

12. Uchiyama, M., and M. Mihara. 1978. Determination of malonaldehyde precursor in tissues by thiobarbituric acid. Anal. Biochem. 86:271-278.

13. Zager, R. A. 1989. Studies of mechanisms and protective maneuvers in myoglobinuric acute renal failure. Lab. Invest. 60:619-629.

14. Gutteridge, J. M., and Y. Y. Hou. 1986. Iron complexes and their reactivity in the bleomycin assay for radical-promoting loosely-bound iron. Free Radical Res. Commun. 2:143-151.

15. Grootveld, M., and B. Halliwell. 1986. Aromatic hydroxylation as a potential measure of hydroxyl-radical formation in vivo: identification of hydroxylated derivatives of salicylate in human body fluids. Biochem. J. 237:499-504.

16. Cao, W., J. M. Carney, A. Duchon, R. A. Floyd, and M. Chevion. 1988. Oxygen free radical involvement in ischemia and reperfusion to brain. Neurosci. Lett. 88:233-238.

17. Floyd, R. A., J. J. Watson, and P. K. Wong. 1984. Sensitive assay of hydroxyl free radical formation utilizing high pressure liquid chromatography with electrochemical detection of phenol and salicylate hydroxylation products. J. Biochem. Biophys. Methods. 10:221-235.

18. Rothgeb, T. M., and F. R. N. Gurd. 1978. Physical methods for the study of myoglobin. Methods Enzymol. 52:473-486.

19. Paller, M. S., and B. E. Hedlund. 1988. Role of iron in postischemic renal injury in the rat. Kidney Int. 34:474-480.

20. Paller, M. S., J. R. Hoidal, and T. F. Ferris. 1984. Oxygen free radicals in ischemic acute renal failure in the rat. J. Clin. Invest. 74:1156-1164.

21. Zager, R. A. 1988. Gentamicin nephrotoxicity in the setting of acute renal hypoperfusion. Am. J. Physiol. 254:F574-F581.

22. Weinberg, J. M., J. A. Davis, M. Abarzua, and T. Kiani. 1990. Glycine-dependent protection of proximal tubules against lethal cell injury due to inhibitors of mitochondrial ATP production. Am. J. Physiol. 258:C1127-C1140.

23. Weinberg, J. M., J. A. Davis, M. Abarzua, R. K. Smith, and R. Kunkel. 1990. Ouabain-induced lethal proximal tubule cell injury is prevented by glycine. Am. J. Physiol. 258:F346-F355.

24. Weinberg, J. M., J. A. Davis, N. F. Roeser, and M. A. Venkatachalam. 1991. Role of increased cytosolic free calcium in the pathogenesis of rabbit proximal tubule cell injury and protection by glycine or acidosis. J. Clin. Invest. $87: 581-590$. 